\title{
DIRECT MEASUREMENT OF THE SIZE OF 2003 UB313 FROM THE HUBBLE SPACE TELESCOPE
}

\author{
M. E. Brown, ${ }^{1}$ E. L. Schaller, ${ }^{1}$ H. G. Roe, ${ }^{1}$ D. L. Rabinowitz, ${ }^{2}$ and C. A. Trujillo ${ }^{3}$ \\ Received 2006 February 8; accepted 2006 April 3; published 2006 May 4
}

\begin{abstract}
We have used the Hubble Space Telescope to directly measure the angular size of the large Kuiper Belt object 2003 UB313. By carefully calibrating the point-spread function of a nearby field star, we measure the size of 2003 UB313 to be $34.3 \pm 1.4$ mas, corresponding to a diameter of $2400 \pm 100 \mathrm{~km}$ or a size $\sim 5 \%$ larger than Pluto. The $V$-band geometric albedo of 2003 UB313 is $86 \% \pm 7 \%$. The extremely high albedo is consistent with the frosty methane spectrum, the lack of red coloring, and the lack of observed photometric variation on the surface of 2003 UB313. Methane photolysis should quickly darken the surface of 2003 UB313, but continuous evaporation and redeposition of surface ices appears capable of maintaining the extreme albedo of this body.

Subject headings: comets: general — infrared: solar system — minor planets, asteroids
\end{abstract}

\section{INTRODUCTION}

The planetary-sized scattered Kuiper Belt object 2003 UB313 was discovered in an ongoing survey at the Samuel Oschin telescope at Palomar Observatory (Brown et al. 2005). The heliocentric distance of $97.5 \mathrm{AU}$ and $V$ magnitude of 18.8 at the time of discovery implied a size larger than Pluto for any albedo lower than $96 \%$, but the initial discovery was incapable of providing a measurement of the albedo. The infrared reflectance spectrum of 2003 UB313 is dominated by absorption due to frozen methane (Brown et al. 2005), like the spectra of Pluto and Triton, suggesting the likelihood of a high albedo for 2003 UB313 like the $50 \%-80 \%$ albedos of these two objects. With such a high albedo, 2003 UB313 would have a diameter in the range of 2500-3200 km.

To date, most Kuiper Belt objects with measured sizes have had their diameters determined through radiometry. In this technique, the measured thermal infrared flux (or fluxes) from an object is converted to a diameter using models in which the thermal emission properties and surface temperature distributions of the object are assumed (Altenhoff et al. 2004; Cruikshank et al. 2005). Owing to the uncertainties in the appropriate model parameters, errors in these size determinations are large. The object (50000) Quaoar had its size directly measured by Hubble Space Telescope (HST) observations (Brown \& Trujillo 2004), which are unaffected by uncertainties in thermal emission modeling. The major source of uncertainties in these measurements is the unknown center-to-limb photometric function of Quaoar, which changes the apparent angular diameter of the disk.

Initial Spitzer Space Telescope observations of 2003 UB313 resulted in a $70 \mu \mathrm{m}$ upper limit of $2 \mathrm{mJy}$, consistent with a size upper limit of $3310 \mathrm{~km}$ and lower limit to the albedo of $44 \%$ (M. E. Brown et al. 2006, in preparation). More recent observations from the Institut de Radioastronomie Millimétrique (IRAM) telescope succeeded in detecting a flux of $1.27 \pm$ $0.29 \mathrm{mJy}$ at $1.2 \mathrm{~mm}$, suggesting a size between 2600 and $3400 \mathrm{~km}$ and an albedo between $42 \%$ and $72 \%$ (Bertoldi et al. 2006). An object of this size would have an angular diameter seen from the Earth of between 37 and 48 mas, similar to or larger than Quaoar, and thus should be directly resolvable from

\footnotetext{
${ }^{1}$ Division of Geological and Planetary Sciences, California Institute of Technology, Pasadena, CA 91125; mbrown@caltech.edu.

${ }^{2}$ Department of Physics, Yale University, New Haven, CT 06520.

${ }^{3}$ Gemini Observatory, 670 North A'ohoku Place, Hilo, HI 96720.
}

$H S T$ observations. We present here such observations and the size and albedo of 2003 UB313 directly measured by HST.

\section{OBSERVATIONS}

We obtained $16119 \mathrm{~s}$ exposures and $12133 \mathrm{~s}$ exposures of 2003 UB313 using the High Resolution Channel (HRC) of the Advanced Camera for Surveys in the F435W filter in two orbits of HST. The first observations began at 8:29 on 2005 December 9 (UT), and one observation was obtained every $170 \mathrm{~s}$. The second observations began at 6:52 on 2005 December 10 and were obtained every $184 \mathrm{~s}$. The timing of the observations was chosen so that 2003 UB313 would be a few arcseconds from a moderately bright field star located at $01^{\mathrm{h}} 35^{\mathrm{m}} 37^{\mathrm{s}} .63,-05^{\circ} 38^{\prime} 57^{\prime \prime} .8$, and the telescope was tracked at the sidereal rate so that accurate pointspread function (PSF) measurements could be obtained from the untrailed image of the star. The motion of 2003 UB313 caused a smearing of about 30 mas of the image of 2003 UB313 during each observation, a factor that needed to be taken carefully into account in the analysis of the angular size of the object.

The star had a brightness of $58.8 \pm 0.2$ counts $\mathrm{s}^{-1}(B=$ $20.152 \pm 0.004)$ in each of the images and was single, well exposed, and not saturated; 2003 UB313 had a brightness of $149.9 \pm 0.7$ counts $\mathrm{s}^{-1}(B=19.136 \pm 0.005)$ and traveled across a field uncontaminated by detectable background determined from our full PSF fitting analysis, described below.

The method used to determine the size of 2003 UB313 from these observations is almost identical to that described and validated in detail in Brown \& Trujillo (2004). Here we very briefly summarize the procedure and particularly discuss a few small improvements. First, for each of the 28 individual stellar images, the PSF is modeled using the HST PSF simulator package TinyTim (Krist \& Hook 2001). While TinyTim's default parameters provide a close match to the average HRC PSF, the HST PSF is not absolutely stable, so a fit is performed to the field star in each image. To obtain a best-fit PSF, we use a downhill simplex $\chi^{2}$ minimization method to fit the first eight Zernike terms in the aberration of the TinyTim model at the location of the star. Figure 1 shows the best-fit focus for the individual images, while Figure 2 gives an example of the bestfit model for one of the images. In Brown \& Trujillo and in subsequent experiments on other images, we found that the focus varied smoothly over the course of an orbit. Here we see the same trend but with a few significant outliers. While some of this scatter is likely real and caused by the increase in jitter with HST now in two-gyro mode, we chose to discard as un- 




Fig. 1.-Top: Best-fit focus (in units of rms waves of aberration at $547 \mathrm{~nm}$ ) for the 28 images. Previous experience has shown that the focus varies smoothly over the course of an orbit. We expect that the large jumps in derived focus are a product of the lower signal-to-noise ratio of the PSF star and of the increased jitter in two-gyro mode. We discard as potentially unreliable all of the measurements marked with an "X" in which the focus appears to shift significantly between images. Bottom: Best-fit disk size for each of the individual images. The mean is shown as the solid line, and the standard deviation is shown as the dashed line.

reliable the images in which the measured focus appears to vary greatly between adjacent observations (although we note that the final results are not sensitive to the removal of these points).

With the aberrations measured for each stellar image, TinyTim is used to construct a PSF at the location of 2003 UB313, accounting for the (small) PSF changes due to the field-dependent aberration of the HRC. This PSF is convolved with the measured motion vector of the object and then convolved with model disks with diameters between 10 and 50 mas. This model image is then convolved with the $3 \times 3$ pixel Gaussian kernel representing the effects of CCD charge diffusion. For each image, a best-fit model disk diameter is found that minimizes the sum of the square of the residuals between the modeled image and the data. An example of a best-fit model is shown in Figure 2.

In this process, the two steps that are the largest source of potential error are the choice of the charge diffusion kernel and the choice of the center-to-limb brightness profile of the model disks. TinyTim provides an estimate of the position-dependent charge diffusion kernel on the HRC CCD, but this estimate is based on extrapolations from measurements at other wavelengths (Krist 2004). Because this parameter is critical, we chose to independently measure the charge diffusion at the location of our star and of 2003 UB313. We used archival images of the globular cluster M92 obtained with HRC in the F435W filter and found several isolated stars near the positions of the central PSF star and the positions of 2003 UB313 on the two separate orbits. We used the procedure described above to fit the PSFs of the globular cluster stars near the location of the central PSF star, and then we transferred this PSF to the locations of 2003 UB313 using TinyTim. We then performed a $\chi^{2}$ minimization of the charge diffusion kernel of the globular cluster stars at the locations of 2003 UB313. This procedure

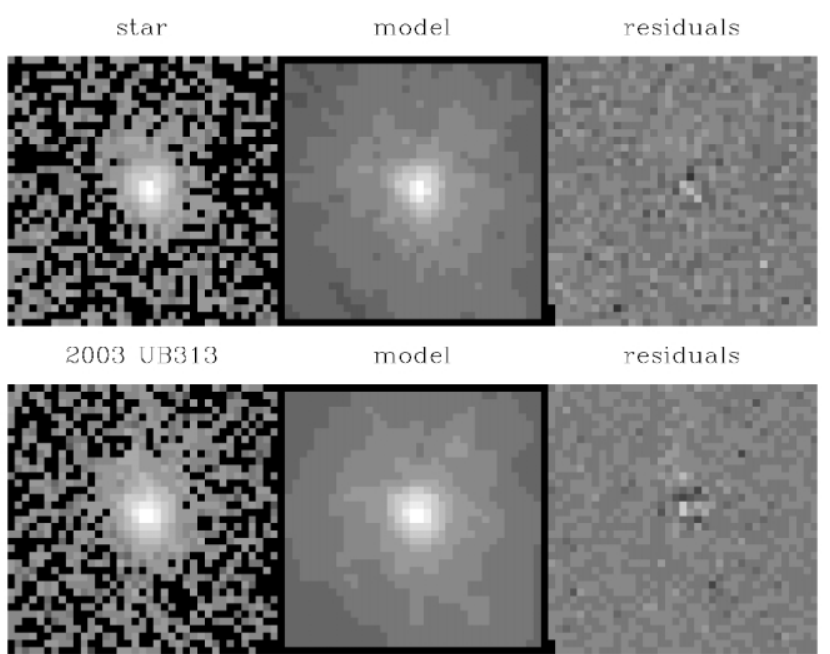

FIG. 2.-Comparison of the star and 2003 UB313 in image 21 with the bestfit models. The data and models share identical logarithmic scales. The residuals are linearly scaled from $\pm 5 \%$ of the maximum of the model image.

does not give us a reliable measure of the absolute width of the charge diffusion kernel (as we have to assume an initial charge diffusion kernel for the central region) but only a measure of the difference between the center location of the PSF star and 2003 UB313. Fortunately, our measurements are only sensitive to relative difference and are not dependent on the absolute values. We find that the Gaussian width of the charge diffusion kernel at the positions of 2003 UB313 are identical for the two measured positions and are $1.04 \pm 0.01$ times larger than the width at the center PSF star.

The other important factor to consider is the center-to-limb brightness profile of the model disks. An object with a flat center-to-limb profile will appear larger than an object whose brightness drops steeply near the limb. In Brown \& Trujillo (2004), we considered an extremely wide range of possible profiles as nothing was known about the surface of Quaoar at the time. For 2003 UB313, however, we know that the surface is covered in methane frost (Brown et al. 2005) like the surfaces of Pluto and Triton. As we have precise measurements of the center-to-limb profile of Triton from well-resolved Voyager measurements, we take this profile, parameterized with a Hapke model (Hillier et al. 1994), as our best analog for the profile of 2003 UB313.

The size measured for 2003 UB313 in each of the 17 images not discarded due to focus deviations is shown in Figure 1. Taking into account the random errors and charge diffusion width uncertainty of 1.0 mas each, we derive an angular size for 2003 $\mathrm{UB} 313$ of $34.3 \pm 1.4$ mas. At a geocentric distance of $96.4 \mathrm{AU}$, this angular diameter corresponds to a diameter of $2400 \pm$ $100 \mathrm{~km}$. For an absolute $V$ magnitude of $H_{v}=-1.12 \pm 0.01$, the albedo of $2003 \mathrm{UB} 313$ is $0.85 \pm 0.07$.

\section{DISCUSSION}

Although 2003 UB313 is $20 \%$ brighter than Pluto in absolute terms, it is only 6\% larger, as the albedo of 2003 UB313 is even higher than that of Pluto. This extremely high derived albedo gives us confidence in our choice of Triton as an analog for the center-to-limb function. Lower albedo icy satellites have center-to-limb profiles that are much flatter than that of Triton. Use of an icy-satellite-like profile would give a smaller size and thus even higher albedo for 2003 UB313, inconsistent with 
the low albedos of the icy satellites. We can, in fact, selfconsistently solve for the center-to-limb profile and the albedo by assuming a Hapke model to give both the profile and the geometric albedo. The profile gives a unique measurement of the angular size, and thus the albedo, but the Hapke model independently gives an albedo. The only Hapke models that give consistent albedos have parameters very similar to Triton, with high single-scattering albedo strongly backscattering particles and smooth surfaces.

The measured size is well below the Spitzer upper limit of $3310 \mathrm{~km}$ but apparently inconsistent with the reported IRAM measured size of $3000 \pm 400 \mathrm{~km}$ (Bertoldi et al. 2006). A reevaluation of the IRAM measurement, however, suggests that the measurements are indeed compatible. The IRAM size measurement assumed an absolute magnitude for 2003 UB313 of $H_{v}=-1.16 \pm 0.1$, which is about $4 \%$ brighter than the best current measurement of $H_{v}=-1.12 \pm 0.01$ (D. L. Rabinowitz et al. 2006, in preparation), suggesting a smaller size. Reevaluating a thermal model with the fainter absolute magnitude and assuming that 2003 UB313 is pole-on and is the largest size allowed by our uncertainties predicts a $1.2 \mathrm{~mm}$ emission of $0.94 \mathrm{mJy}$, consistent at the $1.1 \sigma$ level with the $1.27 \pm 0.29 \mathrm{mJy}$ reported by IRAM. Even an equator-on orientation would give a flux of $0.70 \mathrm{mJy}$, consistent at the $2 \sigma$ level.

The $86 \% \pm 7 \%$ albedo of 2003 UB313 is significantly higher than the $\sim 60 \%$ average of Pluto but consistent with the albedo of the individual brightest areas on Pluto (Young et al. 2001); 2003 UB313 is also less red than Pluto (Brown et al. 2005), and Pluto's darkest regions appear to be a major source of the red color (Young et al. 1999). These two characteristics suggest that the surface of 2003 UB313 might resemble the brightest regions on Pluto while containing few if any of the darkest regions, a suggestion consistent with the lack of detectable photometric variation on 2003 UB313 (H. G. Roe et al. 2006, in preparation).

The geometric albedo of 2003 UB313 is higher than that of all known bodies in the solar system with the exception of Saturn's satellite Enceladus, which has a geometric albedo of $103 \%$ and has active water plumes capable of providing continuous frost resurfacing (Hansen et al. 2006). Even Triton, which has active geysers and appears freshly resurfaced, has a slightly lower albedo of 0.77 (Hillier et al. 1994). Typical inactive water-ice-covered satellites in the outer solar system have albedos ranging from 0.2 to 0.4 , with exceptions only related to activity or exogenic processes. On 2003 UB313, even highly reflective methane frosts will irreversibly darken due to long-term photolysis; some uncommon process that continues to maintain a high albedo on 2003 UB313 is required.

One process unique on large bodies in the Kuiper Belt with eccentric orbits is atmospheric freezeout. At 2003 UB313's $38 \mathrm{AU}$ perihelion equilibrium temperature of $\sim 43 \mathrm{~K}$, the vapor pressure over a solid nitrogen surface would be $\sim 30 \mathrm{~Pa}$, while the pressure over a solid methane surface would be $\sim 0.2 \mathrm{~Pa}$ (Lodders \& Fegley 1998). At the current distance of 97 AU and equilibrium temperature of $27 \mathrm{~K}$, these pressures drop by 6 and 8 orders of magnitude, respectively. Any nitrogen or methane atmosphere that exists at perihelion is essentially completely frozen to the surface by aphelion.

This $560 \mathrm{yr}$ cycle of evaporation and then freezeout of the atmosphere of 2003 UB313 will leave fresh undarkened ices on the top layer at aphelion. A $30 \mathrm{~Pa}$ nitrogen atmosphere at perihelion corresponds to a layer of solid nitrogen several centimeters thick at aphelion, while a $0.2 \mathrm{~Pa}$ methane atmosphere corresponds to a layer tens of microns thick. These layers would sit on top of any dark photolyzed methane and would maintain a high albedo for grain sizes smaller than the surface thickness, which appears likely. At perihelion, the dark areas would be revealed, leading to a Pluto-like appearance. The object 2005 FY9, which also has a surface covered in methane frost (Barkume et al. 2005; Licandro et al. 2006) and is currently at a distance of $52 \mathrm{AU}$, should have a surface temperature approximately midway between Pluto and 2003 UB313. The presence of small but discernible photometric variations on 2005 FY9 (H. G. Roe et al. 2006, in preparation) and the evidence for a small surface coverage of warm dark material (M. E. Brown et al. 2006, in preparation) suggests that 2005 FY9 could have undergone partial atmospheric freezeout, but that at the temperature of 2005 FY9 the process is not complete as on 2003 UB313. The process of atmospheric freezeout, which has long been debated for Pluto (i.e., Stern et al. 1988; Stansberry \& Yelle 1999; Eliot et al. 2004), can now be studied in a growing population of methane-rich objects at a wide range of distances and temperatures in the outer solar system.

We would like to thank the director and staff at the Space Telescope Science Institute (STScI) for providing the opportunity to make these measurements and the assistance in making them happen. This research has been supported by a grant from STScI and from NASA Planetary Astronomy.

\section{REFERENCES}

Altenhoff, W. J., Bertoldi, F., \& Menten, K. M. 2004, A\&A, 415, 771

Barkume, K. M., Brown, M. E., \& Schaller, E. L. 2005, AAS/DPS Meeting, 37, 52.11

Bertoldi, F., Altenhoff, W., Weiss, A., Menten, K. M., \& Thum, C. 2006, Nature, 439, 563

Brown, M. E., \& Trujillo, C. A. 2004, AJ, 127, 2413

Brown, M. E., Trujillo, C. A., \& Rabinowitz, D. L. 2005, ApJ, 635, L97

Cruikshank, D. P., Stansberry, J. A., Emery, J. P., Fernández, Y. R., Werner,

M. W., Trilling, D. E., \& Rieke, G. H. 2005, ApJ, 624, L53

Eliot, J. L., et al. 2004, Nature, 424, 165

Hansen, C. J., et al. 2006, Science, 311, 1422

Hillier, J., Veverka, J., Helfenstein, P., \& Lee, P. 1994, Icarus, 109, 296
Krist, J. E. 2004, Proc. SPIE, 5499, 328

Krist, J. E., \& Hook, R. 2001, The Tiny Tim User's Guide, version 6.0 (Baltimore: STScI)

Licandro, J., Pinilla-Alonso, N., Pedani, M., Oliva, E., Tozzi, G. P., \& Grundy, W. M. 2006, A\&A, 445, L35

Lodders, K., \& Fegley, B. 1998, The Planetary Scientist's Companion (New York: Oxford Univ. Press)

Stansberry, J. A., \& Yelle, R. V. 1999, Icarus, 141, 299

Stern, S. A., Trafton, L. M., \& Gladstone, G. R. 1988, Icarus, 75, 485

Young, E. F., Binzel, R. P., \& Crane, K. 2001, AJ, 121, 552

Young, E. F., Galdamez, K., Buie, M. W., Binzel, R. P., \& Tholen, D. J. 1999, AJ, 117, 1063 